ESAIM: PROCEEDINGS, October 2007, Vol. 22, 40-51

Gabriel Caloz \& Monique Dauge, Editors

\title{
IMAGERIE ÉLECTROMAGNÉTIQUE DE PETITES INHOMOGÉNÉITÉS
}

\author{
Yves CapdeboscQ $^{1}$ and Michael S. Vogelius ${ }^{2}$
}

\begin{abstract}
We give a short review of some recent results concerning asymptotic representation formulas for the perturbations in the electromagnetic fields caused by the presence of small inhomogeneities. We also discuss how these representation formulas may be used to design very effective numerical algorithms to recover the inhomogeneities for overdetermined boundary data.

Résumé. Nous faisons ici une présentation synthétique de certains résultats récents concernant des formules de représentations asymptotiques pour les perturbations des champs électromagnétiques causées par la présences de petite inhomogénéités. Nous discutons aussi de la manière dont ces formules de représentations peuvent être utilisées pour construire des algorithmes numériques très efficaces permettant de reconstruire les inhomogénéités à partir de données au bord sur-déterminées.
\end{abstract}

\section{INTRODUCTION}

Dans cette présentation synthétique, nous allons introduire quelques résultats récents concernant les perturbations des champs électromagnétiques harmoniques en temps causées par la présence d'inhomogénéités de faible volume. Pour simplifier, nous nous limitons au cas dit "transverse magnétique", situation dans laquelle le champ électrique scalaire vérifie une équation de Helmholtz bi-dimensionnelle. Néanmoins, la plupart des résultats présentés pourraient être (ou ont déjà été) obtenus pour les équations de Maxwell générales. Pour commencer, supposons que l'ensemble $\omega_{\epsilon}$ est donné par

$$
\omega_{\epsilon}=\cup_{j=1}^{n}\left(\epsilon B_{j}+\mathbf{x}_{j}\right)
$$

où les $\mathbf{x}_{j}$ sont $n$ points fixés et distincts, à l'intérieur du domaine régulier et borné $\Omega \subset \mathbb{R}^{2}$, et les $B_{j}$ sont $n$ ouverts réguliers et bornés (contenant l'origine). Le paramètre $\epsilon$ est suffisamment petit pour que $\omega_{\epsilon}$ reste à l'intérieur d'un ensemble compact donné $K \subset \Omega$. L'ensemble $\omega_{\epsilon}$ représente les inhomogénéités, et nous supposons que les caractéristiques physiques (la perméabilité magnétique $\mu_{\epsilon}(x)$, et la permittivité électrique $\left.q_{\epsilon}(x)\right)$ sont données par

$$
\mu_{\epsilon}(y)=\left\{\begin{array}{l}
\mu_{1}(x) \text { pour } y \in \omega_{\epsilon} \\
\mu_{0}(x) \text { pour } y \in \Omega \backslash \omega_{\epsilon}
\end{array} \quad, \quad q_{\epsilon}(y)=\left\{\begin{array}{l}
q_{1}(x) \text { pour } y \in \omega_{\epsilon} \\
q_{0}(x) \text { pour } y \in \Omega \backslash \omega_{\epsilon}
\end{array},\right.\right.
$$

où $\mu_{0}, \mu_{1}$ sont des fonctions positives et régulières, et où $q_{0}, q_{1}$ sont des fonctions régulières à parties réelles strictement positives et parties imaginaires positives ou nulles. Le champ électrique (scalaire à valeur complexe)

\footnotetext{
${ }^{1}$ Laboratoire de Mathématiques de Versailles, Université de Versailles Saint-Quentin-en-Yvelines, CNRS, 78035 Versailles cedex, France

2 Department of Mathematics, Rutgers University, New Brunswick, NJ 08903, USA
}

(C) EDP Sciences, SMAI 2007 
est de la forme $e^{-i \lambda t} u_{\epsilon}(x), \lambda>0$, où $u_{\epsilon}$ satisfait

$$
\nabla \cdot\left(\frac{1}{\mu_{\epsilon}} \nabla u_{\epsilon}\right)+\lambda^{2} q_{\epsilon} u_{\epsilon}=0 \quad \text { dans } \Omega,
$$

avec par exemple,

$$
\frac{1}{\mu_{\epsilon}} \frac{\partial u_{\epsilon}}{\partial \nu}=\psi \quad \text { sur } \partial \Omega
$$

où $\partial / \partial \nu$ représente la dérivée normale sortante de $\partial \Omega$. Sous l'hypothèse que la fréquence positive $\lambda$ (ou plutôt, son carré) n'est pas une valeur propre du problème (2)-(3) où les coefficients $\mu_{\epsilon}$ et $q_{\epsilon}$ sont remplacés par $\mu_{0}$ et $q_{0}$, il n'est pas trop difficile de montrer que, pour $\epsilon$ suffisamment petit, le même $\lambda$ n'est pas une valeur propre de (2)-(3) non plus (voir [34]). De plus, si on note par $N_{\lambda}(x, y)$ la solution fondamentale de Neumann, c'est à dire la solution de

$$
\begin{aligned}
\nabla \cdot\left(\frac{1}{\mu_{0}} \nabla N_{\lambda}(\cdot, y)\right)+\lambda^{2} q_{0} N_{\lambda}(\cdot, y) & =\delta_{y} & \text { dans } \Omega, & \text { avec } \\
\frac{1}{\mu_{0}} \frac{\partial}{\partial \nu} N_{\lambda}(\cdot, y) & =0 & \text { sur } \partial \Omega, &
\end{aligned}
$$

pour $y \in \Omega$, et la solution de

$$
\begin{aligned}
\nabla \cdot\left(\frac{1}{\mu_{0}} \nabla N_{\lambda}(\cdot, y)\right)+\lambda^{2} q_{0} N_{\lambda}(\cdot, y) & =0 \quad \text { dans } \Omega, \quad \text { avec } \\
\frac{1}{\mu_{0}} \frac{\partial}{\partial \nu} N_{\lambda}(\cdot, y) & =-\delta_{y} \quad \text { sur } \partial \Omega
\end{aligned}
$$

pour $y \in \partial \Omega$, alors il est possible, en combinant des résultats de [19] et [34], d'obtenir le résultat "perturbatif" suivant.

Proposition 1. Il existe des tenseurs réels, symétriques et définis positifs $M^{(j)}, 1 \leq j \leq n$, tels que

$$
\begin{array}{r}
u_{\epsilon}(y)-u_{0}(y)=\epsilon^{2} \sum_{j=1}^{n}\left(\frac{1}{\mu_{1}\left(\mathbf{x}_{j}\right)}-\frac{1}{\mu_{0}\left(\mathbf{x}_{j}\right)}\right) M^{(j)} \nabla u_{0}\left(\mathbf{x}_{j}\right) \cdot \nabla_{x} N_{\lambda}\left(\mathbf{x}_{j}, y\right) \\
+\epsilon^{2} \lambda^{2} \sum_{j=1}^{n}\left(q_{0}-q_{1}\right)\left(\mathbf{x}_{j}\right)\left|B_{j}\right| u_{0}\left(\mathbf{x}_{j}\right) N_{\lambda}\left(\mathbf{x}_{j}, y\right)+o\left(\epsilon^{2}\right)
\end{array}
$$

pour $y \in \bar{\Omega} \backslash K$.

Les tenseurs $M^{(j)}$ sont calculés de la manière suivante. Pour tout domaine borné et régulier $B$, toute constante $c>0$, et tout $\xi \in \mathbb{R}^{2}$, notons $\phi_{\xi}$ la solution de

$$
\nabla \cdot\left(c^{*} \nabla \phi_{\xi}\right)=0 \quad \text { dans } \mathbb{R}^{2}, \quad \text { avec } \quad \phi_{\xi}(x) \approx \xi \cdot x \quad \text { lorsque } \quad|x| \rightarrow \infty .
$$

où la fonction constante par morceaux $c^{*}$ est donnée par

$$
c^{*}(x)=\left\{\begin{array}{l}
c \text { pour } x \in B \\
1 \text { pour } x \in \mathbb{R}^{2} \backslash B
\end{array}\right.
$$

Le tenseur $2 \times 2 M_{B}[c]$ est défini par son action sur le vecteur $\xi$

$$
M_{B}[c] \xi=\int_{B} \nabla \phi_{\xi}(x) d x .
$$


Il n'est pas difficile de voir que $M_{B}$ est symétrique et définie positive. Le tenseur $M^{(j)}, 1 \leq j \leq n$, qui apparaît dans la Proposition 1 est donné par

$$
M^{(j)}=M_{B_{j}}\left[\frac{\mu_{0}\left(\mathbf{x}_{j}\right)}{\mu_{1}\left(\mathbf{x}_{j}\right)}\right] .
$$

Remarque 1. Le choix de la solution fondamentale qui apparaît dans la Proposition 1 n'est pas essentiel, mais il simplifie l'expression du membre de gauche. Comme cela est expliqué dans remarque 3 de [15] il est possible après des modifications mineures, d'utiliser n'importe quelle solution fondamentale $\Phi_{\lambda}$ qui vérifie

$$
\nabla \cdot\left(\frac{1}{\mu_{0}} \nabla \Phi_{\lambda}(\cdot, y)\right)+\lambda^{2} q_{0} \Phi_{\lambda}(\cdot, y)=\delta_{y} \quad \text { dans un ensemble ouvert contenant } \bar{\Omega} .
$$

Ainsi dans [34] la formule est obtenue, pour $\mu_{0}$ et $q_{0}$ constants (et pour $\left.\mu_{1}\right|_{\epsilon B_{j}+\mathbf{x}_{j}}=\mu_{j},\left.q_{1}\right|_{\epsilon B_{j}+\mathbf{x}_{j}}=q_{j}$ constants) en utilisant la solution fondamentale dans tout l'espace

$$
\Phi_{\lambda}(x, y)=-\mu_{0} \frac{i}{4} H_{0}^{(1)}\left(\lambda \sqrt{\mu_{0} q_{0}}|x-y|\right)
$$

$H_{0}^{(1)}$ étant la fonction de Hankel d'ordre zéro, qui vérifie la condition de radiation sortante. La fomule s'écrit

$$
\begin{aligned}
u_{\epsilon}(y)-u_{0}(y) & -\frac{1}{\mu_{0}} \int_{\partial \Omega}\left(u_{\epsilon}-u_{0}\right)(x) \frac{\partial \Phi_{\lambda}}{\partial \nu_{x}}(x, y) d \sigma_{x} \\
= & \epsilon^{2} \sum_{j=1}^{n}\left(\frac{1}{\mu_{j}}-\frac{1}{\mu_{0}}\right) M^{(j)} \nabla u_{0}\left(\mathbf{x}_{j}\right) \cdot \nabla_{x} \Phi_{\lambda}\left(\mathbf{x}_{j}, y\right) \\
& +\epsilon^{2} \lambda^{2} \sum_{j=1}^{n}\left(q_{0}-q_{j}\right)\left|B_{j}\right| u_{0}\left(\mathbf{x}_{j}\right) \Phi_{\lambda}\left(\mathbf{x}_{j}, y\right)+o\left(\epsilon^{2}\right), y \in \Omega \backslash K .
\end{aligned}
$$

Pour passer à la limite et obtenir une formule valable pour $y \in \partial \Omega$ il faut alors tenir compte de la relation de saut vérifiée par le "potentiel double couche généralisé" $\int_{\partial \Omega}\left(u_{\epsilon}-u_{0}\right)(x) \frac{\partial \Phi_{\lambda}}{\partial \nu_{x}}(x, y) d \sigma_{x}$, c'est-à-dire du fait qu'à la limite lorsque $y$ approche de $\partial \Omega$

$$
\lim \int_{\partial \Omega}\left(u_{\epsilon}-u_{0}\right)(x) \frac{\partial \Phi_{\lambda}}{\partial \nu_{x}}(x, y) d \sigma_{x}=\frac{\mu_{0}}{2}\left(u_{\epsilon}-u_{0}\right)(y)+\int_{\partial \Omega}\left(u_{\epsilon}-u_{0}\right)(x) \frac{\partial \Phi_{\lambda}}{\partial \nu_{x}}(x, y) d \sigma_{x} .
$$

Après un réarrangement on obtient alors

$$
\begin{aligned}
u_{\epsilon}(y)-u_{0}(y) & -\frac{2}{\mu_{0}} \int_{\partial \Omega}\left(u_{\epsilon}-u_{0}\right)(x) \frac{\partial \Phi_{\lambda}}{\partial \nu_{x}}(x, y) d \sigma_{x} \\
=2 & \epsilon^{2} \sum_{j=1}^{n}\left(\frac{1}{\mu_{j}}-\frac{1}{\mu_{0}}\right) M^{(j)} \nabla u_{0}\left(\mathbf{x}_{j}\right) \cdot \nabla_{x} \Phi_{\lambda}\left(\mathbf{x}_{j}, y\right) \\
& +2 \epsilon^{2} \lambda^{2} \sum_{j=1}^{n}\left(q_{0}-q_{j}\right)\left|B_{j}\right| u_{0}\left(\mathbf{x}_{j}\right) \Phi_{\lambda}\left(\mathbf{x}_{j}, y\right)+o\left(\epsilon^{2}\right), \quad y \in \partial \Omega
\end{aligned}
$$

où la densité dans l'intégrale doit être interprétée comme la fonction régulière donnée par $\nabla_{x} \Phi_{\lambda}(x, y) \cdot \nu_{x}$ pour $x \in \partial \Omega \backslash\{y\}$. 
Remarque 2. Concernant les champs électromagnétiques, il est très naturel de considérer le problème dispersif extérieur. Dans ce cas, $\Omega$ est remplacé par $\mathbb{R}^{2}$ tout entier et on décompose le champ "total" $u_{\epsilon}$ en

$$
u_{\epsilon}(y)= \begin{cases}u_{\epsilon}^{(t r)}(y) & \text { pour } y \in \omega_{\epsilon} \\ u_{\epsilon}^{(s)}(y)+u^{(i n c)}(y) & \text { pour } y \in \mathbb{R}^{2} \backslash \omega_{\epsilon}\end{cases}
$$

où le champ réfléchi $u_{\epsilon}^{(s)}$ satisfait la condition de radiation sortante

$$
\frac{\partial}{\partial r} u_{\epsilon}^{(s)}-i \lambda \sqrt{\mu_{0} q_{0}} u_{\epsilon}^{(s)}=o\left(r^{-1 / 2}\right) \quad \text { lorsque } r \rightarrow \infty
$$

en supposant que $\mu_{0}$ et $q_{0}$ sont constants. L'onde incidente $u^{(i n c)}$ est une solution de

$$
\begin{gathered}
\nabla \cdot\left(\frac{1}{\mu_{0}} \nabla u^{(i n c)}\right)+\lambda^{2} q_{0} u^{(i n c)}=0 ; \quad \text { on peut par exemple choisir } \\
u^{(i n c)}(y)=e^{i \lambda \sqrt{\mu_{0} q_{0}} \eta \cdot y} \quad \text { pour un vecteur unitaire } \eta \text { donné, }
\end{gathered}
$$

en supposant à nouveau que $\mu_{0}$ et $q_{0}$ sont constants. La formule de perturbation (6) en devient alors une pour $u_{\epsilon}^{(s)}$ dans le champ lointain, et elle s'écrit

$$
\begin{aligned}
u_{\epsilon}^{(s)}(y)=\epsilon^{2} & \sum_{j=1}^{n}\left(\frac{1}{\mu_{j}}-\frac{1}{\mu_{0}}\right) M^{(j)} \nabla u^{(i n c)}\left(\mathbf{x}_{j}\right) \cdot \nabla_{x} \Phi_{\lambda}\left(\mathbf{x}_{j}, y\right) \\
& +\epsilon^{2} \lambda^{2} \sum_{j=1}^{n}\left(q_{0}-q_{j}\right)\left|B_{j}\right| u^{(i n c)}\left(\mathbf{x}_{j}\right) \Phi_{\lambda}\left(\mathbf{x}_{j}, y\right)+o\left(\epsilon^{2}\right), y \in \mathbb{R}^{2} \backslash K .
\end{aligned}
$$

Cela "découle" immédiatement d'une variante de (6) si l'on prend comme domaine $\Omega$ une boule de rayon suffisamment grand: étant donné que $u_{\epsilon}^{(s)}$ (qui tient lieu de $u_{\epsilon}-u_{0}$ ) n'a pas une dérivée normale qui s'annule sur $\partial \Omega$, une deuxième intégrale de bord, nommément

$$
\frac{1}{\mu_{0}} \int_{\partial \Omega} \frac{\partial u_{\epsilon}^{(s)}}{\partial \nu}(x) \Phi_{\lambda}(x, y) d \sigma_{x}
$$

doit être ajoutée au membre de gauche. En choisissant $\Omega$ suffisamment grand et en intégrant par partie (ou simplement en laissant $\Omega$ approcher $\mathbb{R}^{2}$ tout entier) nous pouvons utiliser le fait qu'à la fois $u_{\epsilon}^{(s)}$ et $\Phi_{\lambda}$ satisfont à la condition de radiation sortante

$$
\frac{\partial v}{\partial r}-i \lambda \sqrt{\mu_{0} q_{0}} v=o\left(r^{-1 / 2}\right) \quad \text { lorsque } r \rightarrow \infty,
$$

pour éliminer les termes sous formes d'intégrales de bord. Le résultat est (8).

Il est bien connu que

$$
H_{0}^{(1)}(r)=\sqrt{\frac{2}{\pi r}} e^{i\left(r-\frac{\pi}{4}\right)}+O\left(r^{-3 / 2}\right), \quad \text { et } \quad\left(H_{0}^{(1)}\right)^{\prime}(r)=\sqrt{\frac{2}{\pi r}} e^{i\left(r+\frac{\pi}{4}\right)}+O\left(r^{-3 / 2}\right)
$$

quand $r \rightarrow \infty$. En conséquence on voit facilement que le membre de droite de (8) est d'ordre $(\epsilon \lambda)^{2} / \sqrt{\lambda}$, et il est effectivement possible de prouver que le reste est $o\left((\epsilon \lambda)^{2} / \sqrt{\lambda}\right)$ pour $\lambda>c_{0}>0$, lorsque $\epsilon \rightarrow 0, \epsilon \lambda \rightarrow 0-$ même 
si $\lambda \rightarrow \infty$. Autrement dit: le développement (8) ( et d'après nous, (6) aussi) donne le vrai terme principal de $u_{\epsilon}^{(s)}$ (ou $\left.u_{\epsilon}-u_{0}\right)$ dès lors que $\lambda>c_{0}$ est $o\left(\epsilon^{-1}\right)$.

Lorsque $\lambda$ atteint $\epsilon^{-1}$ les deux termes du membre de droite de (8) sont tous deux d'ordre $\sqrt{\epsilon}$, alors que pour $\lambda$ plus grand que $\epsilon^{-1}$ ces termes sont asymptotiquement plus grands. Dans le cas radialement symétrique il a été démontré, voir [25], que pour une large classe d'ondes incidentes la norme $L^{2}$ de $u_{\epsilon}^{(s)}$ a une borne supérieure d'ordre $\sqrt{\epsilon}$ uniformément en $\lambda$ - on peut alors conclure qu'un développement tel que (8) (et probablement 6) ne représente pas les termes principaux de $u_{\epsilon}^{(s)}$ (ou $u_{\epsilon}-u_{0}$ ) pour $\lambda$ d'ordre plus grand que $\epsilon^{-1}$.

Bien que beaucoup reste à faire, nous avons obtenu quelques progrès sur la dérivation heuristique de formules qui, dans un ensemble relativement large de directions, et pour de de très grandes fréquences $\lambda$ (d'ordre plus que $\epsilon^{-1}$ ) donnent de bonnes approximations du champ lointain dispersé, voir [26]. Les méthodes utilisées sont un mélange de représentations intégrales, et de développements de type optique géométrique des densités.

\section{LE CAS DE L'ÉLECTROSTATIQUe}

Les formules de perturbations de la section précédente ont toutes des analogues en dimension plus grande que deux, même si elles ne peuvent être vues comme en relations avec des solutions particulières des équations de Maxwell harmoniques en temps. Les versions pour les domaines bornés des formules de perturbations, précisément les formules (4), (6), et (7) font aussi sens dans le cas limite où $\lambda$ vaut zéro. Par exemple l'analogue de (4) dans le cas $m$ dimensionnel, pour $\lambda=0$, établit que

$$
u_{\epsilon}(y)-u_{0}(y)=\epsilon^{m} \sum_{j=1}^{n}\left(\gamma_{1}-\gamma_{0}\right)\left(\mathbf{x}_{j}\right) M^{(j)} \nabla u_{0}\left(\mathbf{x}_{j}\right) \cdot \nabla_{x} N_{0}\left(\mathbf{x}_{j}, y\right)+o\left(\epsilon^{m}\right)
$$

pour $y \in \bar{\Omega} \backslash K$. Ici $\gamma_{0}(x)=1 / \mu_{0}(x), \gamma_{1}(x)=1 / \mu_{1}(x), \gamma_{\epsilon}(x)=1 / \mu_{\epsilon}(x)$, et $u_{\epsilon}$, et $u_{0}$ sont les solutions de

$$
\nabla \cdot\left(\gamma_{\epsilon}(x) \nabla u_{\epsilon}\right)=0 \quad, \quad \text { et } \nabla \cdot\left(\gamma_{0}(x) \nabla u_{0}\right)=0, \quad \text { dans } \Omega,
$$

avec

$$
\gamma_{\epsilon}(x) \frac{\partial}{\partial \nu} u_{\epsilon}=\gamma_{0}(x) \frac{\partial}{\partial \nu} u_{0}=\psi \quad \text { sur } \partial \Omega .,
$$

normalisées par $\int_{\partial \Omega} u_{\epsilon} d \sigma=\int_{\partial \Omega} u_{0} d \sigma=0{ }^{1}$. Le courant prescrit, $\psi$, est supposé être équilibré, dans le sens que $\int_{\partial \Omega} \psi d \sigma=0$. La fonction $N_{0}(\cdot, y)$ résout

$$
\nabla \cdot\left(\gamma_{0} \nabla N_{0}(\cdot, y)\right)=\delta_{y} \quad \text { dans } \Omega, \quad \text { avec } \quad \gamma_{0} \frac{\partial}{\partial \nu} N_{0}(\cdot, y)=\frac{1}{|\partial \Omega|} \quad \text { sur } \partial \Omega
$$

$y \in \Omega$, et

$$
\nabla \cdot\left(\gamma_{0} \nabla N_{0}(\cdot, y)\right)=0 \quad \text { dans } \Omega, \quad \text { avec } \quad \gamma_{0} \frac{\partial}{\partial \nu} N_{0}(\cdot, y)=-\delta_{y}+\frac{1}{|\partial \Omega|} \quad \text { sur } \partial \Omega,
$$

$y \in \partial \Omega$, et est aussi normalisé par $\int_{\partial \Omega} N_{0}(x, y) d \sigma_{x}=0$. $\Omega$ est un domaine borné régulier de $\mathbb{R}^{m}, m \geq 2$. Les tenseurs $M^{(j)}=M_{B_{j}}\left[\frac{\gamma_{1}\left(\mathbf{x}_{j}\right)}{\gamma_{0}\left(\mathbf{x}_{j}\right)}\right]$ sont définis comme précédemment, voir (5). Il est relativement facile de voir que l'expression du tenseur

$$
(c-1) M_{B}[c]
$$

a des limites finies bien définies lorsque $c \rightarrow 0$ et $c \rightarrow \infty$ (voir [19]). Conséquemment, le premier terme du membre de droite de (9) a une limite lorsque $\gamma_{1}\left(\mathbf{x}_{j}\right)$ s'approche de 0 et de $\infty$. Incidemment, cette limite est exactement le terme principal de $u_{\epsilon}^{*}-u_{0}$, où $u_{\epsilon}^{*}$ est l'équivalent de $u_{\epsilon}$ lorsque $\gamma_{1}=0$ ou $\gamma_{1}=\infty$ dans

\footnotetext{
${ }^{1}$ Notons que si l'on interprète $\gamma_{0}$ et $\gamma_{\epsilon}$ comme des conductivités, et $u_{0}, u_{\epsilon}$ comme des potentiels électriques, alors il s'agit de problèmes électrostatiques standards en toute dimension.
} 
$\epsilon B_{j}+\mathbf{x}_{j}[19,24]$. Dans cette ligne nous notons que $\gamma_{1}=0$ dans $\epsilon B_{j}+\mathbf{x}_{j}$ implique la condition au bord $\partial u_{\epsilon}^{*} / \partial \nu=0$ sur $\partial\left(\epsilon B_{j}+\mathbf{x}_{j}\right)$, alors que $\gamma_{1}=\infty$ signifie que $u_{\epsilon}^{*}=$ cste sur $\epsilon B_{j}+\mathbf{x}_{j}$, la constante étant choisie pour que $\int_{\partial\left(\epsilon B_{j}+\mathbf{x}_{j}\right)} \frac{\partial}{\partial \nu} u_{\epsilon}^{*} d \sigma=0$. En d'autres termes, la formule de perturbation à fréquence nulle (9) est valide pour tout $\gamma_{1}$, avec 0 et $\infty$ inclus. Une affirmation du même type n'est pas vraie pour la formule de perturbation plus générale (4) pour l'équation de Helmholtz (fréquence fixe, non nulle) - la preuve la plus simple de cela étant que le terme principal de $u_{\epsilon}^{*}-u_{0}$ correspondant à la condition au bord $u_{\epsilon}^{*}=0 \operatorname{sur} \epsilon B_{j}+\mathbf{x}_{j}$ ( un "obstacle dur") est d'ordre $\epsilon^{m-2}$ pour $m>2$ (et d'ordre $|\log \epsilon|^{-1}$ pour $m=2$ ) pas d'ordre $\epsilon^{m}$ !

Il est possible de généraliser de manière significative la formule perturbation pour $\lambda=0$ (9) pour ce qui concerne la géométrie de l'ensemble $\omega_{\epsilon}$, dans le cas où $\gamma_{0}$ et $\gamma_{1}$ sont tous deux strictement positifs et finis [15-18]. Supposons simplement que $\omega_{\epsilon}$ est une famille d'ensemble mesurables au sens de Lebesgue avec

$$
\left|\omega_{\epsilon}\right|=\operatorname{mes}\left(\omega_{\epsilon}\right) \rightarrow 0
$$

Il est alors possible d'extraire une sous-suite $\omega_{\epsilon_{n}}$, et de définir une mesure de probabilité $\mu$ et une fonction à valeur tensorielle $M(\cdot)$ telle que

$$
\frac{1}{\left|\omega_{\epsilon_{n}}\right|} 1_{\omega_{\epsilon_{n}}} d x \rightarrow d \mu \quad, \quad\left(\gamma_{1}-\gamma_{0}\right) \frac{1}{\left|\omega_{\epsilon_{n}}\right|} 1_{\omega_{\epsilon_{n}}} \nabla u_{\epsilon_{n}} d x \rightarrow\left(\gamma_{1}-\gamma_{0}\right) M(\cdot) \nabla u_{0} d \mu
$$

au sens des mesures. La fonction à valeur tensorielle $M(\cdot)$ vérifie

$$
\begin{gathered}
M_{i j}(x)=M_{j i}(x) \quad, \quad \text { et } \\
\min \left\{1, \frac{\gamma_{0}(x)}{\gamma_{1}(x)}\right\} \leq M(x) \leq \max \left\{1, \frac{\gamma_{0}(x)}{\gamma_{1}(x)}\right\}
\end{gathered}
$$

$\mu$ presque partout dans l'ensemble $\left\{x: \gamma_{0}(x) \neq \gamma_{1}(x)\right\}$.

Suivant la sous-suite $\omega_{\epsilon_{n}}$ nous avons

$$
\begin{gathered}
\left(u_{\epsilon_{n}}-u_{0}\right)(y)=\left|\omega_{\epsilon_{n}}\right| \int_{\Omega}\left(\gamma_{1}-\gamma_{0}\right)(x) M_{i j}(x) \frac{\partial u_{0}}{\partial x_{j}} \frac{\partial N_{0}}{\partial x_{i}}(x, y) d \mu(x) \\
+o\left(\left|\omega_{\epsilon_{n}}\right|\right), \quad y \in \bar{\Omega} \backslash K,
\end{gathered}
$$

pour tout $\psi \in H^{1 / 2}(\partial \Omega)$. La mesure de probabilité $\mu$ et la fonction à valeur tensorielle $M(\cdot)$ dépendra en général de la sous-suite $\omega_{\epsilon_{n}}$ - en d'autre termes, dans cette forme très générale la formule de représentation (11) est un résultat de compacité qui caractérise aussi tous les points limites possibles (au lieu d'un résultat qui garantit l'existence d'une limite unique).

Nous remarquons que dans certains cas particuliers, tels que par exemple lorsque $\omega_{\epsilon}=\cup_{j=1}^{n}\left(\epsilon B_{j}+\mathbf{x}_{j}\right)$ pour des $B_{j}$ et $\mathbf{x}_{j}$ fixés, il existe une limite unique. Une situation similaire (de limite unique) apparaît par exemple lorsque $\omega_{\epsilon}$ prend la forme d'une couche mince de surface moyenne fixée [11]. En général la formule de perturbation (11) n'est pas valable pour $\gamma_{1}$ valant 0 ou $\infty$. L'exemple le plus simple qui montre ceci est le cas d'une couche mince de conductivité zéro ou l'infini, cas dans lequel la limite de $u_{\epsilon}$ lorsque $\left|\omega_{\epsilon}\right|$ s'approche de zéro n'est pas $u_{0}$, mais une solution avec une condition au bord de "fissure" sur la surface moyenne (fixée) de la couche mince. La détermination des cas précis dans lesquels (11) peut être généralisée pour admettre des conductivités extrêmes est le sujet d'un travail indépendant [22].

Les tenseurs de polarisation tels qu'ils sont définis en (5) ont été étudiés depuis longtemps dans la littérature, voir par exemple [27], [30] et [33]. Il existe un lien très naturel entre le tenseur défini en (5) et le tenseur effectif (homogénéisé), $A_{\epsilon}$, obtenu comme mélange des deux matériaux (constants) $\gamma_{0}$ et $\gamma_{1}$ de manière périodique, où $\epsilon B$ représente la part de la cellule de périodicité qui est occupée par $\gamma_{1}$ [10]. Comme cela a été remarqué, par exemple dans [9],

$$
A_{\epsilon}=\gamma_{0}+\theta_{\epsilon}\left(\gamma_{1}-\gamma_{0}\right) \frac{1}{|B|} M_{B}\left[\frac{\gamma_{1}}{\gamma_{0}}\right]+o\left(\theta_{\epsilon}\right)
$$


où $\theta_{\epsilon}$ représente la (faible) fraction volumique de $\epsilon B$ relativement à la cellule de périodicité. En d'autres termes, $M_{B}$ peut être vu comme une "dérivée par rapport à la fraction volumique du tenseur effectif $A_{\epsilon}$ " (remise à l'échelle). En partant des bornes dites de Hashin-Shtrikman pour les valeurs propres du tenseur effectif, [31], et de la relation asymptotique ci-dessus il est alors possible, par des manipulations simples et un passage à la limite $\theta_{\epsilon} \rightarrow 0$, d'obtenir la "borne inférieure" suivante pour les valeurs propres, $\lambda_{i}, 1 \leq i \leq m$, de $\bar{M}_{B}=\frac{1}{|B|} M_{B}\left[\frac{\gamma_{1}}{\gamma_{0}}\right]$ :

$$
\sum_{i=1}^{m} \lambda_{i}^{-1}=\operatorname{Trace}\left(\bar{M}_{B}^{-1}\right) \leq m-1+\frac{\gamma_{1}}{\gamma_{0}}=m a\left(\frac{\gamma_{1}}{\gamma_{0}}\right)
$$

ainsi que la borne supérieure suivante:

$$
\sum_{i=1}^{m} \lambda_{i}=\operatorname{Trace}\left(\bar{M}_{B}\right) \leq m-1+\frac{\gamma_{0}}{\gamma_{1}}=m a\left(\frac{\gamma_{0}}{\gamma_{1}}\right)
$$

Voir par exemple [9] (ou [32]) pour une obtention de la borne inférieure. Il est conjecturé que tout $m$-uplet présent dans le "cube" $\left[\min \left\{1, \frac{\gamma_{0}}{\gamma_{1}}\right\}, \max \left\{1, \frac{\gamma_{0}}{\gamma_{1}}\right\}\right]^{m}$, et vérifiant les deux bornes ci-dessus, peut être obtenu comme ensemble des valeurs propres de $\bar{M}_{B}$ calculé en termes de (5) pour un certain domaine $B$. Voir par exemple [17] pour une construction utilisant des ellipses emboîtées, ou [3] pour une autre construction, qui utilise des domaines simplement connexes $B$, vérifiant cette conjecture en deux dimensions. La Figure 1 représente lensemble des valeurs propres possibles (les deux bornes) dans le cas bi-dimensionnel lorsque $\gamma_{0}<\gamma_{1}$.

Il est remarquable que l'on puisse prouver exactement les mêmes bornes (12) et (13) pour les valeurs propres du tenseur de polarisation généralisé qui apparaît dans la formule asymptotique (11), soit,

$$
\operatorname{Trace}\left(M^{-1}(x)\right) \leq m-1+\frac{\gamma_{1}(x)}{\gamma_{0}(x)}=m a\left(\frac{\gamma_{1}(x)}{\gamma_{0}(x)}\right),
$$

et

$$
\operatorname{Trace}(M(x)) \leq m-1+\frac{\gamma_{0}(x)}{\gamma_{1}(x)}=m a\left(\frac{\gamma_{0}(x)}{\gamma_{1}(x)}\right),
$$

$\mu$ presque partout dans l'ensemble $\left\{x: \gamma_{0}(x) \neq \gamma_{1}(x)\right\}$. Pour le calcul de ces deux bornes nous renvoyons à [18] (voir aussi [17]). Si $\gamma_{0}$ et $\gamma_{1}$ sont constants, avec $\gamma_{0} \neq \gamma_{1}$, alors il existe des borne analogues pour $\int_{\Omega} M(x) d \mu$ :

$$
\operatorname{Trace}\left(\int_{\Omega} M(x) d \mu\right)^{-1} \leq m-1+\frac{\gamma_{1}}{\gamma_{0}}=m a\left(\frac{\gamma_{1}}{\gamma_{0}}\right),
$$

et

$$
\operatorname{Trace}\left(\int_{\Omega} M(x) d \mu\right) \leq m-1+\frac{\gamma_{0}}{\gamma_{1}}=m a\left(\frac{\gamma_{0}}{\gamma_{1}}\right) .
$$

La borne (17) découle immédiatement de (15), par intégration. Pour l'obtention de (16) voir [17].

\section{ReConstruction DiRECTE En PRATiQue}

Les formules de représentation présentées dans les deux sections précédentes peuvent être utilisées efficacement dans le cadre d'algorithmes de reconstruction. Elles peuvent être utilisées de deux manières différentes, que nous allons maintenant détailler dans le cas de la fréquence nulle : (A1) par le calcul du terme asymptotique dominant de l'énergie correspondante, et par l'utilisation des bornes (10), et (16)-(17), il est possible d'obtenir des bornes très efficaces du volume total $\left|\omega_{\epsilon}\right|$ des inhomogénéités, et (A2) par l'utilisation de la nature particulière des solutions dipolaires qui forment fonctionnellement une base pour la "réponse électrostatique aux 


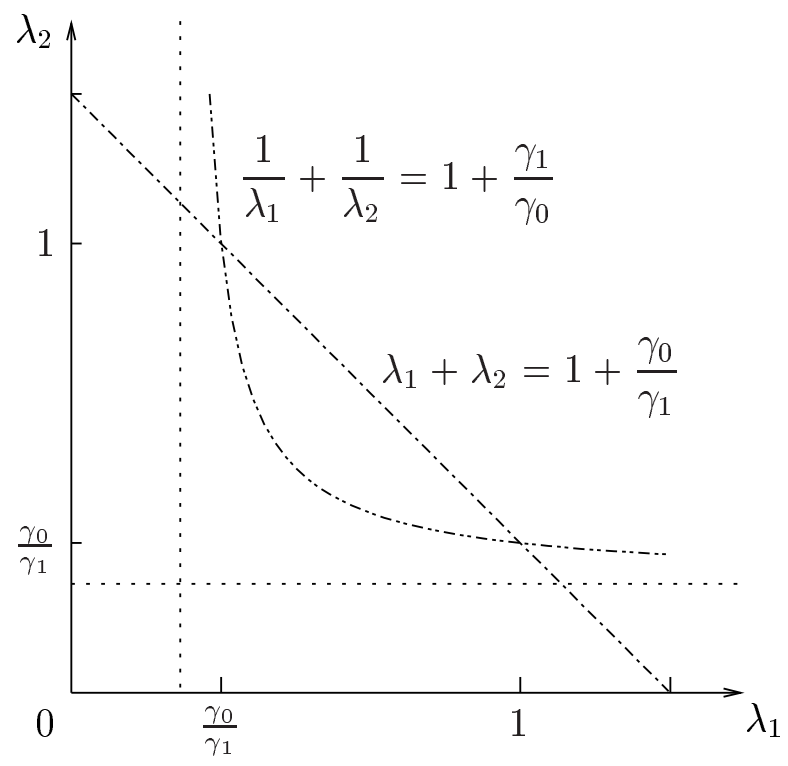

FiguRE 1. Valeurs propres possibles pour le tenseur de polarisation $M_{i j}$, apparaissant dans (9), dans le cas $m=2$ (et $\gamma_{0}<\gamma_{1}$ ), suivant la caractérisation (14) et (15).

perturbations", il est possible, dans certain cas, de construire des algorithmes directs permettant de trouver le support de la mesure $\mu$.

L'application (A1) a globalement été développée dans [16] et [17]. Nous en montrons un "instantané". Soit $w$ une fonction $\gamma_{0}$-harmonique régulière arbitraire, c'est-à-dire, une solution régulière de $\nabla \cdot\left(\gamma_{0} \nabla w\right)=0$ dans $\Omega$. Notons tout d'abord que, en terme de la fonction de Neumann $N_{0}$, et du flux normal à la frontière $\gamma_{0} \frac{\partial w}{\partial \nu}$, on a

$$
\frac{\partial w}{\partial x_{i}}(x)=-\int_{\partial \Omega} \frac{\partial N_{0}}{\partial x_{i}}(x, y) \gamma_{0} \frac{\partial w}{\partial \nu}(y) d \sigma_{y}
$$

En multipliant la formule de représentation (11) par $\gamma_{0} \frac{\partial w}{\partial \nu}$ et en intégrant sur $\partial \Omega$ on obtient ainsi

$$
\begin{aligned}
\int_{\partial \Omega}\left(u_{\epsilon_{n}}-u_{0}\right) \gamma_{0} \frac{\partial w}{\partial \nu} d \sigma & \\
= & -\left|\omega_{\epsilon_{n}}\right| \int_{\Omega}\left(\gamma_{1}-\gamma_{0}\right) M_{i j}(x) \frac{\partial u_{0}}{\partial x_{j}}(x) \frac{\partial w}{\partial x_{i}}(x) d \mu+o\left(\left|\omega_{\epsilon_{n}}\right|\right) .
\end{aligned}
$$

Supposons maintenant que $\gamma_{0}$ et $\gamma_{1}$ sont des constantes, avec $\gamma_{0} \neq \gamma_{1}$. Considérons les flux normaux au bord $\psi^{(i)}=\gamma_{0} \nu_{i}, 1 \leq i \leq m$, et les potentiels correspondant $u_{\epsilon}^{(i)}$ et $u_{0}^{(i)}=x_{i}+$ cste, et définissons la matrice symétrique $m \times m, D$, de "données mesurées" suivantes

$$
D_{i j}=\int_{\partial \Omega}\left(u_{\epsilon_{n}}^{(i)}-u_{0}^{(i)}\right) \gamma_{0} \frac{\partial u_{0}^{(j)}}{\partial \nu} d \sigma=\gamma_{0} \int_{\partial \Omega}\left(u_{\epsilon_{n}}^{(i)}-x_{i}\right) \nu_{j} d \sigma \quad, \quad 1 \leq i, j \leq m
$$


A partir de (18) et (19) il vient que la matrice des données mesurées et le tenseur de polarisation moyen sont reliés par

$$
\begin{aligned}
D_{i j} & =-\left|\omega_{\epsilon_{n}}\right| \int_{\Omega}\left(\gamma_{1}-\gamma_{0}\right) M_{k l}(x) \frac{\partial u_{0}^{(i)}}{\partial x_{l}}(x) \frac{\partial u_{0}^{(j)}}{\partial x_{k}}(x) d \mu+o\left(\left|\omega_{\epsilon_{n}}\right|\right) \\
& =\left|\omega_{\epsilon_{n}}\right|\left(\gamma_{0}-\gamma_{1}\right) \int_{\Omega} M_{i j} d \mu+o\left(\left|\omega_{\epsilon_{n}}\right|\right) .
\end{aligned}
$$

En conséquence

et

$$
\left|\omega_{\epsilon_{n}}\right|(1+o(1))=\frac{|\operatorname{Trace} D|}{\left|\gamma_{0}-\gamma_{1}\right| \operatorname{Trace}\left(\int_{\Omega} M d \mu\right)}
$$

$$
\left|\omega_{\epsilon_{n}}\right|(1+o(1))=\frac{\operatorname{Trace}\left(\int_{\Omega} M d \mu\right)^{-1}}{\left|\gamma_{0}-\gamma_{1}\right|\left|\operatorname{Trace} D^{-1}\right|} .
$$

A partir de (20) et des bornes

$$
\min \left(1, \frac{\gamma_{0}}{\gamma_{1}}\right) \leq \int_{\Omega} M_{j j} d \mu \leq \max \left(1, \frac{\gamma_{0}}{\gamma_{1}}\right)
$$

(voir (10) ) on obtient immédiatement que

$$
\frac{\left|D_{j j}\right|}{\left|\gamma_{0}-\gamma_{1}\right|} \min \left(1, \frac{\gamma_{1}}{\gamma_{0}}\right) \leq\left|\omega_{\epsilon_{n}}\right|(1+o(1)) \leq \frac{\left|D_{j j}\right|}{\left|\gamma_{0}-\gamma_{1}\right|} \max \left(1, \frac{\gamma_{1}}{\gamma_{0}}\right)
$$

$1 \leq j \leq m$. Ce sont les estimations pour une seule mesure obtenues dans [16].

A partir de (21), (22) et des bornes pour $\operatorname{Trace}\left(\int_{\Omega} M d \mu\right)$ et $\operatorname{Trace}\left(\int_{\Omega} M d \mu\right)^{-1}$, données dans (16)-(17), nous concluons que

$\operatorname{avec} h(s)=\left(a\left(s^{-1}\right)\right)^{-1}$, et

$$
\frac{\mid \text { Trace } D \mid}{m\left|\gamma_{0}-\gamma_{1}\right|} h\left(\frac{\gamma_{1}}{\gamma_{0}}\right) \leq\left|\omega_{\epsilon_{n}}\right|(1+o(1))
$$

$$
\left|\omega_{\epsilon_{n}}\right|(1+o(1)) \leq \frac{m}{\left|\gamma_{0}-\gamma_{1}\right|\left|\operatorname{Trace}\left(D^{-1}\right)\right|} a\left(\frac{\gamma_{1}}{\gamma_{0}}\right) .
$$

La borne inférieure (24) est la même que la borne inférieure à $m$-mesures trouvée dans [16], la borne supérieure (25) est plus précise que la borne supérieure $m$-mesures obtenue dans [16] (et elle dépend des toutes les $(m+$ 1) $m / 2$ mesures $D_{i j}, 1 \leq i \leq j \leq m$ ). En fait la borne supérieure $m$-mesures obtenue dans [16]

$$
\left|\omega_{\epsilon_{n}}\right|(1+o(1)) \leq \frac{\mid \text { Trace } D \mid}{m\left|\gamma_{0}-\gamma_{1}\right|} a\left(\frac{\gamma_{1}}{\gamma_{0}}\right)
$$

est une conséquence immédiate de (25) en remarquant que

$$
\frac{1}{\mid \text { Trace }\left(D^{-1}\right) \mid} \leq \frac{\mid \text { Trace } D \mid}{m^{2}} .
$$

On remarque que les bornes (24)-(26) sont asymptotiques dans le sens qu'elles ne sont vraies qu'à la limite lorsque $\left|\omega_{\epsilon}\right|$ s'approche de 0. La même remarque devrait a priori s'appliquer aux bornes (23), cependant, comme cela a été vérifié dans [1], [2], ces estimations plus larges sont en fait valables indépendamment de la taille de $\left|\omega_{\epsilon}\right|$. Nous avons une expérience numérique significative dans l'utilisation des bornes (24)-(26), et elles 
sont en pratique de très bons estimateurs du volume réel, même lorsque la fraction volumique des inclusions atteint 10\% - 20\%. Les bornes présentées ici correspondent à une fréquence fixée ( 0 ) - il serait intéressant d' obtenir des raffinements utilisant plusieurs (ou une bande complète de) fréquences, et d'évaluer la pertinence numérique de ces bornes.

Les algorithmes qui ont été développés pour l'application (A2) ont de nombreux éléments en communs avec les méthodes MUSIC ou d'échantillonage linéaire (linear sampling), voir [12, 20,21,23, 29]. Nous revenons rapidement sur l'algorithme développé dans [14]. Supposons que $\gamma_{0}$ et $\gamma_{1}$ sont des constantes, avec $\gamma_{0} \neq \gamma_{1}$, et supposons que l'ensemble $\omega_{\epsilon}$ est de la forme

$$
\omega_{\epsilon}=\cup_{j=1}^{n}\left(\epsilon B_{j}+\mathbf{x}_{j}\right)
$$

où les $B_{j}$ sont des domaines bornés et réguliers, et où $\epsilon \ll 1$. Dans ce cas la mesure de probabilité limite $\mu$ de (11) devient

$$
\mu=\sum_{j=1}^{n} \frac{\left|B_{j}\right|}{\sum_{k=1}^{n}\left|B_{k}\right|} \delta_{\mathbf{x}_{j}},
$$

et la fonction à valeur dans les tenseurs de polarisation $M(\cdot)$ est seulement définie aux points $\mathbf{x}_{j}, 1 \leq j \leq n$, avec $M\left(\mathbf{x}_{j}\right)$ donné par

$$
M\left(\mathbf{x}_{j}\right)=\frac{1}{\left|B_{j}\right|} M_{B_{j}}\left[\frac{\gamma_{1}}{\gamma_{0}}\right],
$$

où $M_{B_{j}}[c]$ est telle que définie en $(5)$ dans la première section.

La formule de représentation pour $u_{\epsilon}-u_{0}$ s'ecrit alors

$$
\left(u_{\epsilon}-u_{0}\right)(y)=\epsilon^{m} \sum_{j=1}^{n} \tilde{M}_{k l}^{(j)} \frac{\partial u_{0}}{\partial x_{l}}\left(\mathbf{x}_{j}\right) \frac{\partial N_{0}}{\partial x_{k}}\left(\mathbf{x}_{j}, y\right)+o\left(\epsilon^{m}\right)
$$

où le terme de reste $o\left(\epsilon^{m}\right)$ est en fait $O\left(\epsilon^{m+1 / 2}\right)$ (cf. [19]). Ici $\tilde{M}^{(j)}$ représente le tenseur de polarization remis à l'échelle de manière appropriée $\left(\tilde{M}^{(j)}=\left(\gamma_{1}-\gamma_{0}\right)\left|B_{j}\right| M\left(\mathbf{x}_{j}\right)\right)$. Comme cela a été remarqué auparavant, cette formule ne demande pas l'extraction d'une sous-suite $\epsilon_{n}$ pour être valide (la limite est unique). En terme d'application "Neumann-à-Dirichlet" $\Lambda_{\epsilon}$ et $\Lambda_{0}$ (correspondant aux conductivités $\gamma_{\epsilon}$ et $\gamma_{0}$ ) cette formule de représentation peut être écrite

$$
\left(\Lambda_{\epsilon}-\Lambda_{0}\right) \psi=\epsilon^{m} \mathfrak{D} \psi+o\left(\epsilon^{m}\right),
$$

avec $\mathfrak{D}: L^{2}(\partial \Omega) \cap\left\{\psi: \int_{\partial \Omega} \psi=0\right\}=L_{0}^{2}(\partial \Omega) \rightarrow L_{0}^{2}(\partial \Omega)$ représentant l'opérateur linéaire borné, de rang fini, auto-adjoint,

$$
\mathfrak{D} \psi(y)=\sum_{j=1}^{n} \tilde{M}_{k l}^{(j)} \frac{\partial u_{0}}{\partial x_{l}}\left(\mathbf{x}_{j}\right) \frac{\partial N_{0}}{\partial x_{k}}\left(\mathbf{x}_{j}, y\right),
$$

(on se rappelle que $u_{0}$ dépend continûment et linéairement de $\psi$ ). Soit $d \in \mathbb{R}^{m} \backslash\{0\}$ un vecteur donné. Pour tout $z \in \Omega$ on définit la fonction $g_{z} \in C^{\infty}(\partial \Omega)$ par

$$
g_{z}(\cdot)=d \cdot \nabla_{x} N_{0}(z, \cdot)=d \cdot \nabla_{y} N_{0}(\cdot, z)
$$

Une preuve du résultat suivant est donnée dans [14].

Lemme 1. Soient les fonctions $g_{z}(\cdot), z \in \Omega$, définies comme ci-dessus, et soient $\left\{\mathbf{x}_{j}\right\}_{j=1}^{n}$ les "centres" des différentes inhomogénéités de $\omega_{\epsilon}=\cup_{j=1}^{n}\left(\epsilon B_{j}+\mathbf{x}_{j}\right)$. Alors

$$
z \in\left\{\mathbf{x}_{j}\right\}_{j=1}^{n} \quad \text { si et seulement si } \quad g_{z} \in \mathfrak{D}\left(L_{0}^{2}(\partial \Omega)\right) \text {. }
$$


Ce lemme est le fondement d'un algorithme numérique très efficace pour l'identification de l'ensemble des "centres" $\left\{\mathbf{x}_{j}\right\}_{j=1}^{n}$ :

(a) On calcule tout d'abord la décomposition spectrale d'une version discrétisée (mesurée) de la différence "Neumann-à-Dirichlet" $\Lambda_{\epsilon}-\Lambda_{0}$. En prenant l'espace vectoriel engendré par toutes les valeurs propres au dessus d'une certaine limite, on obtient une approximation de l'image de $\Lambda_{\epsilon}-\Lambda_{0}$. Grâce à l'hypothèse de petitesse de $\epsilon$, cela donne aussi une approximation de l'image par l'opérateur $\mathfrak{D}$ de $L_{0}^{2}(\partial \Omega)$.

(b) Soit $V_{k}$ l'espace vectoriel engendré par les $k$ premiers vecteurs propres calculés en (a) (ceux correspondant aux valeurs propres les plus grandes), et soit $P_{k}$ la projection $L^{2}$ sur $V_{k}$. Prenons alors $z$ comme les points d'un treillis fin couvrant $\Omega$, et calculons

$$
\cot \theta_{k}(z)=\frac{\left\|P_{k} g_{z}\right\|}{\left\|\left(I-P_{k}\right) g_{z}\right\|}
$$

la "cotangente" de l'angle entre la fonction $g_{z}$ et l'approximation de l'image de $\mathfrak{D}$ (engendrée par les $k$ vecteurs propres). En calculant cette "cotangente" pour quelques valeurs de $k$, ou, pour peu qu'on ait une idée du nombre des inhomogénéités, en faisant ce calcul seulement pour $k=n$ (ou proche de $n$ ). Les points où la "cotangente" prend de grandes valeurs sont interprétés comme des endroits très vraisemblablement dans le support de $\mu$.

Pour les expériences numériques utilisant cet algorithmes (ou d'autres très sembables) voir [13], [14] et [6]. La méthode décrite ici peut être facilement étendue à une fréquence non nulle et au système complet de Maxwell [4]. Il serait ici encore intéressant de voir si il est possible d'améliorer la reconstruction en utilisant plusieurs (ou une bande complète de) fréquences. Cette méthode a aussi été appliquée avec succès dans des situations où les données (sur-déterminées ou de Cauchy) sont seulement disponibles sur une partie de la frontière, et dans des situations où les inhomogénéités sont sur ou près de la partie inaccessible de la frontière (voir [6]). Une question très intéressante dans la pratique concerne la manière dont l'application Neumann-à-Dirichlet continue est approchée par un opérateur de rang fini - en d'autres termes : y a-t-il des méthodes particulièrement judicieuses pour le faire, en vue d'améliorer la précision de la méthode directe ci-dessus. L'implémentation dans [6] emploie de manière très efficace les traces de solutions dipolaires pour construire une telle approximation.

\section{REMERCIEMENTS}

Les recherches d'Y. Capdeboscq sont partiellement financées par le projet de ANR EchoScan. Les recherches de M.S. Vogelius sont partiellement financées par NSF grant DMS-0604999. Cet article représente un résumé d'une conférence plénière donnée par le second auteur au 38ième Congrès National d'Analyse Numérique qui s'est tenu à Guidel en Mai 2006. Il souhaite remercier l'Équipe d'analyse numérique de l'IRMAR pour l'avoir invité à cet évènement très bien organisé.

\section{REFERENCES}

[1] G. Alessandrini, A. Morassi, and E. Rosset, Size estimates. Inverse Problems: Theory and Applications, eds. G. Alessandrini and G. Uhlmann. Contemporary Mathematics, 333 (2003), pp. 1-33.

[2] G. Alessandrini, E. Rosset, and J.K. Seo, Optimal size estimates for the inverse conductivity problem with one measurement. Proceedings of the American Mathematical Society 128 (2000) pp. 53-64.

[3] H. Ammari, Y. Capdeboscq, H. Kang, E. Kim, and M. Lim, Attainability by simply connected domains of optimal bounds for the polarization tensor. European J. Appl. Math., 17 (2006), pp. 201-219.

[4] H. Ammari, E. Iakovleva, and D. Lesselier, Two numerical methods for recovering small inclusions from the scattering amplitude at a fixed frequency. SIAM J. Sci. Comput., 27 (2005), pp. 130-158.

[5] H. Ammari, and H. Kang, Reconstruction of Small Inhomogeneities from Boundary Measurements. Lecture Notes in Mathematics, 1846, Springer-Verlag, 2004.

[6] H. Ammari, H. Kang, E. Kim, K. Louati, and M.S. Vogelius, A MUSIC-type algorithm for detecting internal corrosion from electrostatic boundary measurements. Rapport Interne, Rutgers University, Mars 2007.

[7] H. Ammari, S. Moskow, and M.S. Vogelius, Boundary integral formulae for the reconstruction of electric and electromagnetic inhomogeneities of small volume. ESAIM: Cont. Opt. Calc. Var., 9 (2003), pp. 49-66. 
[8] G. Bal, Optical tomography for small volume absorbing inclusions. Inverse Problems, 19 (2003), pp. 371-386.

[9] A.Yu. Belyaev, and S.M. Kozlov, Hierarchical structures and estimates for homogenized coefficients. Russian J. Math. Phys., 1 (1992), pp. 3-15.

[10] A. Bensoussan, J.-L. Lions, and G. Papanicolaou, Asymptotic Analysis for Periodic Structures. North-Holland, Amsterdam, 1978.

[11] E. Beretta, E. Francini, and M.S. Vogelius, Asymptotic formulas for steady state voltage potentials in the presence of thin inhomogeneities. A rigorous error analysis. Journal Mathematiques Pures et Appliquees, 82 (2003), pp. 1277-1301.

[12] J.G. Berryman, L. Borcea. G.C. Papanicolaou, and C. Tsogka, Statistical stability and time-reversal imaging in random media. Geometric Methods in Inverse Problems and PDE Control, eds. C.B. Croke, I. Lasiecka, G. Uhlmann, and M.S. Vogelius. IMA Volumes in Mathematics and its Applications, 137 (2003), pp. 15-24.

[13] M. Brühl and M. Hanke, Numerical implementation of two noniterative methods for locating inclusions by impedance tomography. Inverse Problems, 16 (2000), pp. 1029-1042.

[14] M. Brühl, M. Hanke, and M.S. Vogelius, A direct impedance tomography algorithm for locating small inhomogeneities. Numerische Mathematik, 93 (2003), pp. 635-654.

[15] Y. Capdeboscq and M.S. Vogelius, A general representation formula for boundary voltage perturbations caused by internal conductivity inhomogeneities of low volume fraction. M2AN, 37 (2003), pp. 159-173.

[16] Y. Capdeboscq and M.S. Vogelius, Optimal asymptotic estimates for the volume of internal inhomogeneities in terms of multiple boundary measurements. M2AN, 37 (2003), pp. 227-240.

[17] Y. Capdeboscq and M.S. Vogelius, A review of some recent work on impedance imaging for inhomogeneities of low volume fraction. Contemporary Mathematics 362, eds. C. Conca, R. Manasevich, G. Uhlmann, and M.S. Vogelius, pp. 69-88. AMS, Providence, 2004.

[18] Y. Capdeboscq and M.S. Vogelius, Pointwise polarization tensor bounds and applications to voltage perturbations caused by thin inhomogeneities. Asymptotic Analysis, 50 (2006), pp. 175-204.

[19] D.J. Cedio-Fengya, S. Moskow, and M.S. Vogelius, Identification of conductivity imperfections of small diameter by boundary measurements. Continuous dependence and computational reconstruction. Inverse Problems 14 (1998) pp. 553-595.

[20] M. Cheney, The linear sampling method and the MUSIC algorithm. Inverse Problems, 17 (2001), pp. 591-595.

[21] D. Colton and A. Kirsch, A simple method for solving inverse scattering problems in the resonance region Inverse Problems, 12 (1996), pp. 383-393.

[22] F. de Gournay, and M.S. Vogelius. En préparation.

[23] A.J. Devaney, Super-resolution processing of multi-static data using time reversel and MUSIC. Submitted to IEEE Trans. Image Process.

[24] A. Friedman, and M.S. Vogelius, Identification of small inhomogeneities of extreme conductivity by boundary measurements: a theorem on continuous dependence. Arch. Rat. Mech. Anal., 105 (1989), pp. 299-326.

[25] D.J. Hansen, C. Poignard, and M.S. Vogelius, Asymptotically precise norm estimates of scattering from a small circular inhomogeneity. Applicable Analysis, à paraître 2007.

[26] D.J. Hansen, and M.S. Vogelius, High frequency perturbation formulas for the effect of small inhomogeneities. Rapport Interne, Rutgers University, Janvier 2006.

[27] D.S. Jones, Acoustic and Electromagnetic Waves. Oxford University Press, 1986.

[28] H. Kang, J.K. Seo, and D. Sheen, The inverse conductivity problem with one measurement: stability and estimation of size. SIAM J. Math. Anal., 28 (1997), pp. 1389-1405.

[29] A. Kirsch, The MUSIC algorithm and the factorization method in inverse scattering theory for inhomogeneous media. Inverse Problems, 18 (2002), pp. 1025-1040.

[30] R.E. Kleinman, and T.B.A. Senior, Rayleigh scattering. Chapter in Low and High Frequency Asymptotics, eds. V.K. Varadan and V.V. Varadan. Elsevier, Amsterdam, 1986.

[31] R.V. Kohn, and G.W. Milton, On bounding the effective conductivity of anisotropic composites. Homogenization and Effective Moduli of Materials and Media, eds. J.L. Ericksen, D. Kinderlehrer, R.V. Kohn, and J.-L. Lions. IMA Vol. Math. Appl., 1 (1986), pp. 97-125.

[32] R. Lipton, Inequalities for electric and elastic polarization tensors with applications to random composites. J. Mech. Phys. Solids, 41 (1993), pp. 809-833.

[33] M. Schiffer, and G. Szëgo, Virtual mass and polarization Trans. Am. Math. Soc., 67 (1949), pp. 130-205.

[34] M.S. Vogelius, and D. Volkov, Asymptotic formulas for perturbations in the electromagnetic fields due to the presence of inhomogeneities of small diameter. M2AN, 34 (2000), pp. 723-748. 